Original

\title{
Desarrollo y validación del primer cuestionario español de calidad de vida en pacientes con cáncer de próstata, basado en la percepción del paciente
}

\author{
Francisco Gómez Veiga ${ }^{1}$, José M. Cozar Olmo², Stefan Günthner ${ }^{3}$, Miquel Puyol Pallas ${ }^{4}$, \\ Ángel Silmi Moyano ${ }^{5}$ Eva Baró Ramos ${ }^{6}$, Francisco J. Rebollo ${ }^{7}$ \\ ${ }^{1}$ Complejo Hospitalario Universitario, A Coruña; ${ }^{2}$ Hospital Virgen de las Nieves, Granada, ${ }^{3}$ Hospital Clinico de \\ Valencia. ${ }^{4}$ Hospital Sant Joan de Déu, Martorell (Barcelona), ${ }^{5}$ Hospital Clinico San Carlos, Madrid; ${ }^{6} 3 D$-Health \\ Research. ${ }^{7}$ Abbott Laboratories S.A. España
}

\section{Resumen}

Introducción: Asegurar calidad de vida relacionada con la salud(CVRS) durante la supervivencia del paciente con cáncer de próstata (CaP) es primordial. El desarrollo de cuestionarios específicos de CVRS facilita evaluar el impacto de la enfermedad, de su tratamiento y aspectos de percepción del paciente (preocupaciones, expectativas e intereses) que podrían pasar desapercibidos. Objetivos: Desarrollar y validar el primer cuestionario español de CVRS específico para pacientes con CaP.

Materiales y métodos: El cuestionario CAVIPRES tuvo 3 fases de desarrollo: 1-generación de ítems (investigación documental, sesión de expertos y 2 grupos de discusión con pacientes), 2-reducción de ítems (encuesta auto administrada con el precuestionario y análisis clinimétrico y psicométrico), 3: validación del cuestionario (estudio observacional con 2 entrevistas al paciente que completaba los tests CAVIPRES, SF-36 y UCLA-PCI y análisis de propiedades psicométricas en términos de validez, fiabilidad y sensibilidad al cambio).

Resultados: La fase 1 generó un precuestionario de 75 potenciales ítems. En la fase 2, 412 pacientes respondieron a la versión preliminar y se excluyeron 35 ítems. En la fase 3, 436 pacientes respondieron a la versión prevalidada. Se obtuvo un cuestionario de 30 ítems para investigación clínica (CAVIPRES-30). El CAVIPRES-30 mostró viabilidad (ítems perdidos <4\%), factibilidad (efecto techo: $0 \%$ y efecto suelo: $0 \%$ en la puntuación global), alta fiabilidad en cuanto a consistencia interna (alfa $=0,77)$ y a fiabilidad re-test $(\mathrm{CCI}=0,92)$. Hubo una buena correlación entre CAVIPRES y los tests SGF-36 y UCLA-PCI para dimensiones semejantes.

Conclusión: El cuestionario CAVIPRES-30 es un instrumento factible, fiable y sensible al cambio, útil en la investigación clínica del cáncer de próstata.

Palabras clave: Cuestionario. Validez. Factibilidad. Fiabilidad. Calidad de vida. Cáncer de próstata.

\section{Development and validation of the first spanish questionnaire of quality of life in patients with prostate cancer, based on the patient's perception}

\section{Abstract}

Background: To ensure health related quality of life (HRQOL) during the survival of patients with prostate cancer (PCa) is a priority. The development of specific HRQOL questionnaires aids to assess the disease impact, its treatment and the patient's perception (concerns, expectations and interests) that might go unnoticed.

Objectives: To develop and validate the first Spanish HRQL questionnaire specific for patients with PCa.

Materials and methods: The questionnaire CAVIPRES had 3 phases of development:1.-generation of items (literature research, expert meeting and 2 focus groups with patients), 2.- reduction of items (self-administered survey with a preliminary version and clinimetric and psychometric analyses), 3: validation of the questionnaire (observational study with 2 visits where patients completed the CAVIPRES, SF-36 and UCLA-PCI tests and analysis of psychometric properties in terms of validity, reliability and sensitivity to change).

Results: A preliminary list of 75 potential items was obtained in phase 1.412 patients were recruited and answered this preliminary version in phase 2. 35 items were deleted. 436 patients responded to the definitive-30-ítems obtained version (CAVIPRES-30) in Phase 3. CAVIPRES-30 showed feasibility (items lost $<4 \%$, ceiling effect: $0 \%$ floor effect: $0 \%$ in the overall score), high reliability in terms of internal consistency (alpha $=0.77$ ) and re-test reliability $(\mathrm{ICC}=0.92)$. There was a good correlation between tests CAVIPRES and SGF-36 and UCLA-PCI for similar domains

Conclusion: The questionnaire CAVIPRES-30 is a feasible, reliable and sensitive to change, useful in clinical research for prostate cancer.

Keywords: Questionnaire. Validity. Feasibility. Reliability. Quality of life. Prostate cancer. 
T os avances en uro-oncología han permitido que muchos pacientes con cáncer de próstata (CaP) vivan más tiempo. Este incremento en "cantidad" de vida debe asociarse ineludiblemente a "calidad" de vida, siendo un objetivo principal para los profesionales médicos.

La concepción actual de "enfermedad" contempla mejorar aspectos clínicos del paciente pero también físicos, emocionales y sociales. Este pensamiento aplicado al manejo terapéutico, determina que en los estudios de pacientes con CaP, se tenga en consideración componentes de bienestar global y de calidad de vida específica relacionada con la enfermedad y con su tratamiento ${ }^{1}$.

Quien mejor puede responder sobre su calidad de vida es el propio paciente, por tanto, la CVRS es un parámetro objetivable mediante la entrevista al paciente. A pesar de que muchos estudios ${ }^{2,3}$ demuestran que el paciente oncológico prefiere cualquier tratamiento a la sensación de que "no se hace nada" o acepta tratamientos muy agresivos aún con bajas expectativas de beneficio, lo cierto es que se empieza a disponer de resultados que apuntan en otro sentido. En un estudio con pacientes con CaP localizado, adecuadamente informados, el $75 \%$ elegía tandas de radioterapia a dosis inferiores apostando por una mejora en la calidad de vida ${ }^{4}$.

Los cánceres urológicos conllevan disfunción sexual, incontinencia urinaria y/o trastornos intestinales por extensión del cáncer o, como efecto secundario a los tratamientos quirúrgico, radioterápico u hormonal. Estas disfunciones impactan profundamente en la calidad de vida del paciente. Es lógico pues, que se subraye el interés en estudios sobre la calidad de vida de los pacientes con $\mathrm{CaP}^{5,6}$.

Actualmente se dispone de instrumentos específicos para la evaluación de la calidad de vida en pacientes con CaP. El cuestionario UCLA-Prostate Cancer Index (UCLA-PCI) ${ }^{7}$ mide la afectación urinaria, sexual e intestinal en pacientes con CaP y se completó con aspectos generales de calidad de vida integrando el 36-Item Short-Form Health Survey $(\mathrm{SF}-36)^{8}$ originando el cuestionario RAND SF-36v2 ${ }^{9}$. El EPIC(Expanded Prostate Cancer Index) ${ }^{10}$ es otro cuestionario que valora el impacto de los tratamientos en la calidad de vida del paciente con CaP.

También hay dos cuestionarios genéricos en los que se desarrolló un módulo específico para el CaP: el Functional Assessment of Cancer TherapyGeneral (FACT-G) que adicionó 12 ítems originando el FACT-P ${ }^{11}$ y European Organisation for Research and Treatment of Cancer Quality of Life Questionnaire (EORTC QLQ) ${ }^{12}$ al que se añadió un módulo con 25 ítems originando el EORTC QLQ PR-25 ${ }^{13}$.

Los anteriores cuestionarios fueron elaborados en EEUU, Canadá o Inglaterra y algunos han sido validados al español ${ }^{14,15}$. El uso de cuestionarios fuera de sus países de origen exige una adaptación cultural seguida de un depurado análisis psicométrico para validar la nueva versión lingüística ${ }^{16}$. Estos procedimientos, si bien pueden obtener el cuestionario validado con relativa rapidez, no siempre garantizan que su adaptación a la nueva población abarque todos los aspectos sociales y la idiosincrasia del nuevo entorno de aplicación ${ }^{17}$. Lo ideal es crear un instrumento autóctono, cuyo desarrollo pueda explorar la manera de cubrir todas las esferas sensibles de impactar en la calidad de vida del paciente, así como aprovechar la coyuntura para aportar ciertas mejorías (adecuar la extensión o afinar propiedades psicométricas).

El proyecto CAVIPRES (Cuestionario Español de Calidad de Vida en Pacientes con Cáncer de Próstata) se concibió para desarrollar y validar un cuestionario español de calidad de vida en cáncer de próstata, basado en la percepción del paciente.

\section{MATERIAL Y MÉTODOS}

La creación del cuestionario CAVIPRES-30 tuvo tres fases de desarrollo que se exponen a continuación (Fig. 1).

\section{Fase I: generación de items}

Se realizó entre Septiembre y Noviembre de 2006. Consistió en una investigación documental, una sesión de expertos y 2 grupos de discusión con pacientes.

Se consultó la base de datos MEDLINE, y se usó buscadores electrónicos generales (Google, etc), especializados (QOLID - Quality of Life Instrument Database) y por palabras clave. La revisión exhaustiva de la literatura se centró en instrumentos psicométricos de CVRS específicos para pacientes con $\mathrm{CaP}$, para hallar potenciales fuentes de información en el desarrollo de ítems.

La sesión dirigida de expertos contó con urólogos y expertos en psicometría. Se llevó a cabo una moderación grupal por el método de metaplan, garantizando la participación equitativa de los asistentes, combinando técnicas de "brain-storming" y usando técnicas de consenso para establecer prioridades. 


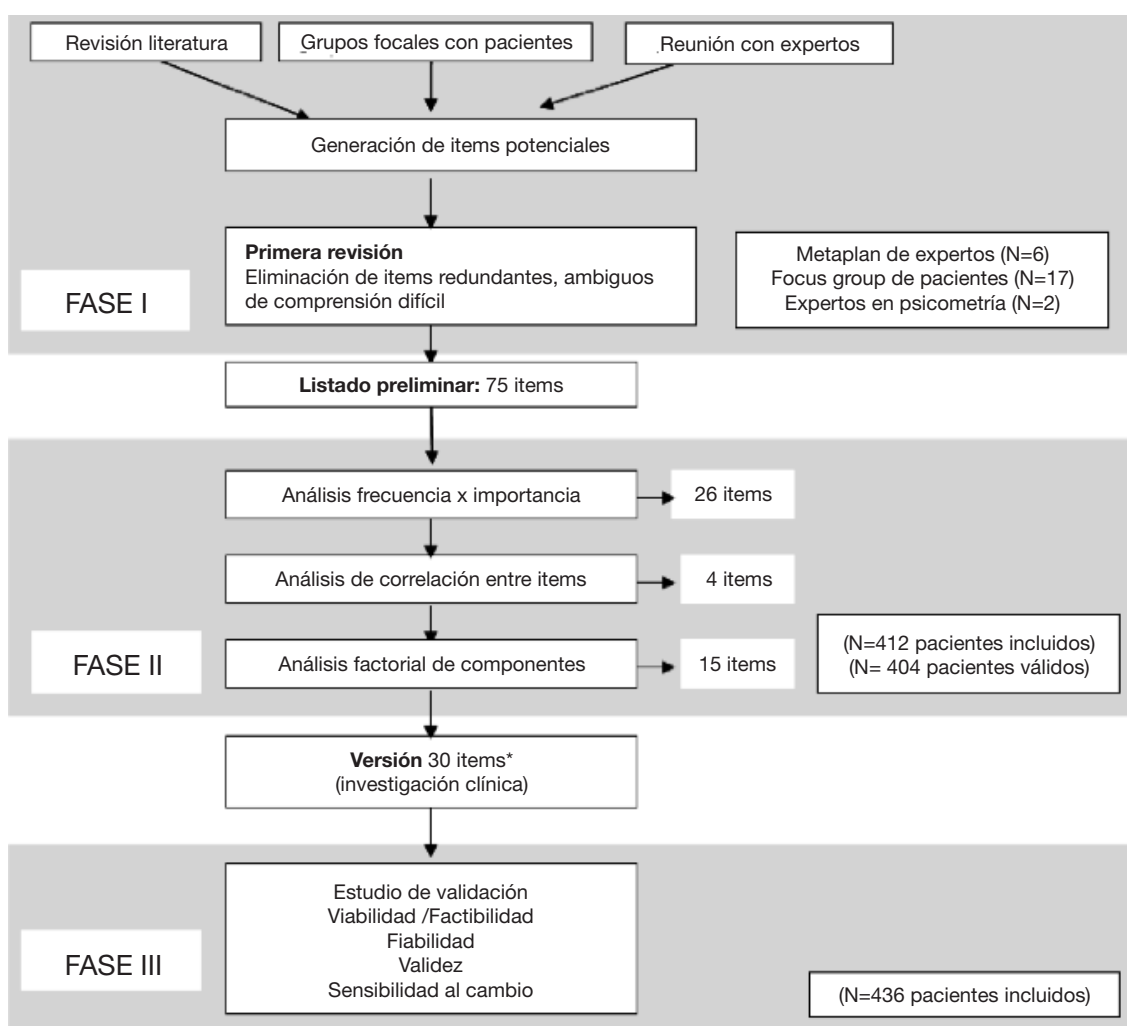

FIGURA 1. Descripción de los items excluidos en durante las diferentes etapas del proceso de desarrollo y validación del cuestionario CAVIPRES.

\section{Fase II: Reducción de items}

Se realizó un estudio multicéntrico epidemiológico y transversal, con la participación de 103 especialistas en urología en atención ambulatoria, procedentes de centros sanitarios distribuidos por toda la geografía española.

El reclutamiento de pacientes y el trabajo de campo se realizaron entre febrero y mayo de 2007. Tras otorgar su consentimiento por escrito, fueron considerados candidatos a participar pacientes mayores de edad, con diagnóstico confirmado de $\mathrm{CaP}$, en cualquier estadio de la enfermedad, sin otro proceso oncológico concomitante y que no estuvieran simultáneamente participando en otro estudio de investigación clínica.

Se diseñaron dos cuadernos de recogida de datos (CRDs) específicamente para este estudio. En el cuaderno del investigador, completado por el urólogo, se registraron datos

La sesión de metaplan perfiló aspectos formales para construir un instrumento práctico y útil, definió factores de calidad de vida percibida por el paciente con $\mathrm{CaP}$ e indicadores con valor clínico para la medición de resultados en salud.

Las reuniones de discusión con pacientes con CaP se organizaron como grupos focales, reclutando una muestra heterogénea según características sociodemográficas (edad, nivel de estudios, situación familiar) y clínicas (estadio de la enfermedad, etc.), para asegurar la variabilidad de la población diana. Los pacientes, debidamente informados, consintieron en participar y no se guardó sus señas identificativas en ningún archivo ni documento de la sesión.

Las sesiones de discusión fueron grabadas con audio-casetes para preservar palabras o expresiones de sus asistentes que podian ser relevantes en la generación de ítems. La transcripción de contenidos, analizada por 2 expertos en psicometría, generó un listado inicial de ítems potenciales. La selección de los ítems desechó aquellos que resultaron ambiguos, de difícil compresión, redundantes o irrelevantes. Se obtuvo un listado inicial de 75 items que conformó un precuestionario. sociodemográficos (edad, estatus conyugal, nivel de formación, situación laboral y origen geográfico) y características clínicas (tiempo de evolución del cáncer, gradación de Gleason, estratificación TNM, estado de actividad ECOG, pauta actual de tratamiento, sintomatología y antecedentes patológicos de interés).

El cuaderno del paciente con el precuestionario era autoadministrado, a ser posible en una sola sesión (o en sesiones consecutivas espaciadas entre sí un máximo de un día).

Los datos obtenidos fueron evaluados bajo un enfoque analítico clinimétrico y psicométrico que permitió una reducción del conjunto inicial de ítems hasta llegar a una versión pre-validada de 20 ítems (CAVIPRES-20) para uso en la práctica clínica y a la versión del 30 items (CAVIPRES-30) para uso en la investigación clínica que se presenta en esta comunicación.

\section{Fase III: Evaluación de las propiedades psicométricas del cuestionario}

Se llevó a cabo un estudio epidemiológico observacional prospectivo con un periodo de seguimiento del paciente de 3 meses para analizar las propiedades psicométricas del CAVIPRES-30 en una muestra de la población diana. 
Participaron 292 investigadores, especialistas en urología, procedentes de centros hospitalarios públicos distribuidos por toda España. Los pacientes que dieron su consentimiento firmado para ser entrevistados cumplían los criterios de selección comunes a la fase II de la validación del cuestionario.

Se registraron los datos de los pacientes en 2 ocasiones en 2 CRDs específicos para el estudio. El CRD del investigador contenía las mismas variables sociodemográficas y clínicas ya descritas en la fase II y, además, recogía la opinión del investigador.

El CRD del paciente contenía el cuestionario RAND SF-36v2 estructurado en 28 preguntas. Las 11 primeras contienen los 36 ítems del SF_36, validado en español ${ }^{18}$. Las 17 siguientes preguntas corresponden al UCLA-PCI (7) y contiene sus 20 ítems. El UCLA-PCI dispone de reconocidas adaptaciones transculturales al español ${ }^{19} \mathrm{y}$ distribuye sus ítems en 6 escalas que miden función y grado de molestias a nivel urinario, intestinal y sexual. También contenía el cuestionario CAVIPRES-30. 24 de sus ítems se distribuyen en 4 dimensiones que abordan aspectos psicológicos, expectativas del paciente, apoyo social y de pareja y vida sexual. Los 6 items restantes requieren la opinión del encuestado sobre su enfermedad. Las 4 dimensiones contienen preguntas con opciones de respuesta en escala tipo Likert de 5 puntos ( $1=$ "siempre" y $5=$ "nunca"). En el CRD del paciente se registraba el tiempo necesario y la valoración de la facilidad para completar el CAVIPRES-30.

Los 2 cuestionarios se pasaban en dos controles. Antes de iniciar el segundo control el paciente respondía a una pregunta "ad-hoc" para evaluar el cambio global del estado de salud ("En estos últimos 3 meses, ¿cree que su estado de salud es..?, con 13 posibles respuestas ordenadas en $1=$ "ha mejorado muchísimo", 13= "ha empeorado muchísimo")

\section{ANÁLISIS DE DATOS Y PROCEDIMIENTOS ESTADÍSTICOS}

\section{Reducción de items}

La reducción de ítems implicó una serie de pasos:

1. análisis de respuestas pérdidas con exclusión de aquellos pacientes que no hubieran respondido más del $20 \%$ de los ítems

2. análisis de "frecuencia $\mathrm{x}$ producto" $\left(\mathrm{F}^{*} \mathrm{P}\right)$ para valorar la importancia de los ítems (considerándose aptos para la versión final del cuestionario aquellos ítems que el encuestado valoraba con mayor frecuencia o con mayor importancia/relevancia) ${ }^{20,21}$.

3. análisis de correlaciones para eliminar redundancias por exclusión del ítem de menor importancia $\left(\mathrm{F}^{*} \mathrm{P}\right)$ cuando dos ítems mostraban una correlación de Pearson superior a 0,8 ,

4. análisis de correlación entre ítem y escala con exclusión de aquellos ítems con baja correlación con la escala (índice de homogeneidad $<0,20$ ).

Los ítems restantes se analizaron para conocer la estructura subyacente de la escala. Se usó el análisis de la validez factorial por el método de componentes principales, con rotación de factores por el método ortogonal (varimax) y se condicionó su selección a aquellos que tuvieran valor eigen superior a 1,0. Para conocer la confiabilidad del cuestionario y valorar consistencia interna y redundancia ${ }^{20}$ se aplicó el alfa de Cronbach correlacionando cada ítem con su dimensión y con el total de la escala.

Se estimó necesaria una muestra de por lo menos 400 pacientes con CaP para la fase de reducción de ítems, suponiendo que la aplicación de los diversos criterios analíticos lograría una reducción hasta 30-40 ítems y que se requería un mínimo de 10 pacientes por ítem para garantizar la aplicabilidad de las técnicas estadísticas multivariadas de análisis factorial sobre los ítems finales ${ }^{20,21}$.

Los datos de la población fueron analizados de forma descriptiva y de acuerdo a los análisis psicométricos expuestos.

\section{VALIDACIÓN DEL CUESTIONARIO CAVIPRESS}

Se analizó descriptivamente la muestra. La viabilidad de CAVIPRES-30 se analizó calculando el porcentaje de respuestas perdidas por ítem y por dimensiones, el tiempo medio de respuesta y la facilidad de uso, según la distribución de la población en la respuesta de estas variables.

La consistencia interna global y por dimensiones se evaluó mediante el alfa de Cronbach, aceptando que una fiabilidad superior a 0,80 era suficiente para su uso agregado. La fiabilidad test-retest se evaluó en el cuestionario global y por dimensiones analizando la asociación entre las puntuaciones obtenidas en los dos controles y la respuesta a la pregunta ad-hoc sobre cambios en el estado de salud entre ambos controles mediante el coeficiente de correlación intraclase y considerando aceptables valores $^{3} 0,70^{20}$. 
La validez convergente se analizó estimando los coeficientes de Pearson entre CAVIPRES y los cuestionarios UCLA-PCI y SF-36. Se esperaban correlaciones más elevadas entre CAVIPRES y el cuestionario UCLA-PCI que entre CAVIPRES-30 y el cuestionario genérico de calidad de vida SF-36.

La sensibilidad al cambio se evaluó calculando el tamaño del efecto obteniendo un índice de la diferencia de la puntuación del cuestionario entre los dos controles dividida por la desviación estándar de la visita basal. Este índice se calculó para aquellos pacientes que refirieron mejoría y cuya repuesta a la pregunta "ad-hoc" oscilaba entre haber mejorado "bastante" y "muchísimo". El tamaño del efecto se cuantificó como "efecto pequeño" $(0,2-0,5)$, "efecto moderado" $(0,5-0,8)$ y efecto grande" $(>0,8)^{22}$.

Para evaluar la fiabilidad, validez y sensibilidad al cambio de CAVIPRES-30 y comparar sus características psicométricas con otros cuestionarios validados, se estimó necesaria una muestra de aproximadamente 450 pacientes con CaP (150 sujetos garantizaba la validación de la fiabilidad test-retest y 300 sujetos garantizaba validación de la sensibilidad al cambio y la especificidad del instrumento). Se asumió que este tamaño de muestra (o inferior si mostraba buenas cualidades psicométricas) permitiría evaluar las propiedades psicométricas del cuestionario con una potencia del $80 \%$, asumiendo un error alfa de 0,05.

\section{RESULTADOS}

\section{Generación de items}

La búsqueda bibliográfica identificó 2 cuestionarios (RAND SF-36v2 y EPIC) diseñados para su aplicación en pacientes con CaP. De estos test se seleccionaron items que, potencialmente, podrían ser incorporados al nuevo cuestionario, con sus adaptaciones social, culturales y/o psicométricas pertinentes.

En la sesión de discusión con expertos participaron 5 urólogos y un experto en psicometría. La sesión facilitó el establecimiento del marco conceptual del cuestionario y de sus características formales. Este abordaje con expertos facilitó el diseño de un guión semiestructurado para coordinar las reuniones de discusión con pacientes.

Se realizaron 2 grupos focales con pacientes, procedentes de consultas externas de Urología de 2 centros hospitalarios de Valencia y Barcelona, con la participación de 8 y 9 pacientes respectivamente. Estas reuniones fueron determinantes para la obtención de 75 ítems que conformaron el precuestionario.

\section{Obtención del número definitivo de ítems del cuestionario}

La fase de reducción de ítems (ver figura 1) se basó en un estudio epidemiológico en el que se reclutó una muestra de 412 pacientes con cáncer de próstata cuyas características sociodemográficas y clínicas se describen en la Tabla 1.

$\mathrm{El}$ análisis de reducción de ítems por cómputo del índice frecuencia *importancia, excluyó los 26 items con menor impacto. El análisis por casos excluyó 5 pacientes que presentaban más del 20\% de ítems sin respuesta y 1 paciente por datos incoherentes.

Sobre los 49 ítems restantes, se analizó las correlaciones entre ítems excluyendo 4 ítems adicionales por estar fuertemente correlacionados entre sí (Correlación de Pearson, coeficiente $>0,8$ ).

Se realizaron diversos análisis factoriales con objetivo exploratorio, para confirmar las posibles dimensiones del cuestionario. Tras valorar la aportación de cada ítem al alfa de Cronbach de la dimensión a la que pertenecía y su contribución a la varianza total, se eliminaron 15 ítems.

El análisis factorial confirmatorio de 5 dimensiones para los 30 ítems restantes presentaba una alfa de Cronbach de 0,80 y explicaba en un 55,3\% la varianza total. Esto permitió establecer el cuestionario definitivo conformado en 5 dimensiones:

- Aspectos psicológicos (ítems 1-8)

- Esperanza y futuro (ítems 9-12)

- Apoyo social y pareja (ítems 13-18)

- Vida sexual (ítems 19-24)

- Información y comunicación (ítems 25-30)

La Tabla 2 muestra la correlación entre cada ítem y su dimensión y cada dimensión con el global del cuestionario. La correlación ítem-puntuación total obtuvo una alfa de Cronbach con un rango entre 0,604 y 0,884 , a excepción de 1 ítem con alfa de 0,577 .

\section{Valoración psicométrica de CAVIPRES-30}

436 pacientes de $\mathrm{CaP}$ distribuidos por toda España (Fig. 2) participaron en la validación del cuestionario. Sus características sociodemográficas y clínicas se describen en la Tabla 1.

En términos de factibilidad, el porcentaje de respuestas perdidas (sin respuesta) para 26 items fue inferior a $1,2 \%$. Los ítems contenidos en la subescala Aspectos psicológicos mostraron un porcentaje de pérdidas entre el 0,5 y 0,9\%. Entre los ítems vincu- 
Tabla 1. Características basales de los dos estudios de campo del proyecto: reducción de ítems y validación

\begin{tabular}{lrr}
\hline Variables & $\begin{array}{r}\text { Fase 2 } \\
\text { Reducción de ítems }\end{array}$ & $\begin{array}{r}\text { Fase 3: } \\
\text { Validación }\end{array}$ \\
\hline $\mathbf{N}$ & 412 & 436 \\
Edad, media (DE) & $72(8,8)$ & $71,5(7,6)$ \\
Estado civil, N(\%) & & \\
Soltero & $18(4,4 \%)$ & $17(3,9 \%)$ \\
Casado/Pareja & $343(83,2 \%)$ & $352(80,7 \%)$ \\
Separado & $6(1,5 \%)$ & $15(3,4 \%)$ \\
Divorciado & $4(0,97 \%)$ & $2(0,5 \%)$ \\
Otro & $40(9,7 \%)$ & $50(11,5 \%)$
\end{tabular}

Nivel de estudios, $\mathrm{N}(\%)$

No sabe leer ni escribir

Sabe leer y/o escribir, sin estudios

Estudios primarios

Estudios secundarios

Estudios universitarios

Tiempo desde el diagnóstico primario, media (DE)

Con sintomatologia previa al diagnóstico, $\mathrm{N}(\%)$

Gradación histológica de Gleason, N(\%)

$$
\begin{array}{r}
2 \\
3 \\
4 \\
5 \\
6 \\
7 \\
8 \\
9 \\
10
\end{array}
$$

ECOG, N(\%)

Vida normal sin limitación

Limitación a grandes esfuerzos

Precisa ayuda en la mayoría actividades

$>50 \%$ encamado

Encamado todo el tiempo

Extensión de la enfermedad, N(\%)

Metástasis

Locorregional

Regional

No clasificables

Intención del tratamiento neoplásico, $\mathrm{N}(\%)$

Pacientes en tratamiento

$$
\begin{aligned}
& \text { Adyuvante } \\
& \text { Paliativo } \\
& \text { Curativo }
\end{aligned}
$$

$35(13,4 \%)$

$156(59,8 \%)$

$69(26,4 \%)$

$82(24 \%)$

$175(51,2 \%)$

$96(28,1 \%)$

Tipo de tratamiento, $\mathrm{N}(\%)$

Pacientes en tratamiento

Conducta expectante

Guimioterapia

Radioterapia

Hormonoterapia

Cirugia

Otros

Tipo de cirugia, N(\%)

Prostatectomía radical

Resección transuretral

Criocirugía

Otro lados a Esperanza y Futuro hubo un porcentaje de respuesta perdidas entre 0,2 y $0,7 \%$. Los ítems de Vida Sexual mostraron un porcentaje de pérdidas entre el 0,7 y $1,1 \%$. Se detectó entre un 2,3 y $4,4 \%$ de respuestas perdidas en los items de Apoyo social y pareja. Los ítems relacionados con Comunicación e información mostraron entre un 0,5 y un $0,9 \%$ de respuestas perdidas. $\mathrm{El}$ promedio de ítems perdidos por paciente encuestado fue de 0,36 (DS: 1,64).

En la Tabla 3 se muestran los resultados del análisis de viabilidad mostrando el "efecto suelo" y el "efecto techo", la consistencia interna y la fiabilidad test-retest. El "efecto techo" fue inferior al 8\% para las máximas puntuaciones de todas las dimensiones, mientras que el "efecto suelo" se dio en menos de un $2 \%$ de las mínimas puntuaciones en todas las dimensiones, exceptuando la dimensión Vida Sexual que presentó un efecto suelo en un $31,8 \%$ de los casos con mínimas puntuaciones. Más del 65\% de los encuestados empleaban un tiempo no superior a 20 minutos en completar el cuestionario. Casi el 69\% de los pacientes consideró que el cuestionario era más bien "fácil" de responder, mientras que un $14 \%$ lo consideró más o menos "difícil" de responder.

El análisis de la fiabilidad mostró que el cuestionario presentaba una consistencia interna muy satisfactoria (alfa de Cronbach $>0,7$ ), de acuerdo con el alfa obtenida para la puntuación global y para cada una de las dimensiones (Tabla 3). 
Tabla 2. Resultados del análisis de consistencia de los 30 ítems definitivos. Correlación ítem- dimensión y dimensión-total (Alfa de Cronbach)

\begin{tabular}{|c|c|c|}
\hline Dimensiones & Ítems & Alpha \\
\hline Aspectos psicológicos & 8 & 0,8659 \\
\hline P14. Mi enfermedad me preocupa & & 0,8455 \\
\hline P19. Mi enfermedad me impide hacer una vida normal & & 0,8587 \\
\hline P20. Pienso en mi enfermedad & & 0,8355 \\
\hline P21. Necesito hablar de las preocupaciones o miedos que me causa mi enfermedad. & & 0,8506 \\
\hline P22. Me preocupa cómo evolucionará mi enfermedad & & 0,847 \\
\hline P23. Me preocupa encontrarme peor & & 0,8468 \\
\hline P24. Mi enfermedad afecta negativamente a mi vida & & 0,8443 \\
\hline P29. Me molesta que me consideren un enfermo de cáncer & & 0,868 \\
\hline
\end{tabular}

Esperanza y futuro

P15. Vivo el presente con ilusión

P16. Veo el futuro con optimismo

P17. A pesar de mi enfermedad, soy capaz de disfrutar de la vida

P18. Mi enfermedad hace que aprecie más algunas cosas de la vida

\section{Apoyo social y pareja}

P1. La familia me ayuda con mi enfermedad y su tratamiento

P3. A pesar de la enfermedad y sus consecuencias, me siento unido a mi pareja

P5. Aunque mi pareja me comprende, a mi me sigue preocupando mi problema de erección

P6. Mi pareja me ayuda y colabora para solucionar mis problemas sexuales

P9. Me siento entendido y apoyado por mis amigos

P56. Los profesionales sanitarios me apoyan respecto a mi problema de impotencia

\section{Vida sexual}

P54. Tengo problemas de erección

P57. Tengo problemas para alcanzar el orgasmo

P58. Tengo problemas para eyacular

P43. Siento que mi vida sexual se ha acabado debido a mi enfermedad

P48. He perdido el interés por el sexo a causa de mi enfermedad

$\mathrm{P} 55$. Me quedo a medias' en mis relaciones sexuales

\section{Comunicación e información}

P69. En general, creo que necesito más información sobre mi enfermedad

P71. En general, creo que debería haber recibido más información sobre secuelas

P72. Me gustaría que hubiera un servicio de apoyo para las personas que tenemos esta enfermedad

P73. He tenido la necesidad de hablar de mi enfermedad con alguien

P74. Creo que intercambiar experiencias con otras personas me daría un poco más de confianza

P75. Me gustaría poder estar a solas con mi médico para preguntarle todas mis dudas
4

\section{$\mathbf{0 , 8 4 1 3}$}

0,7853

0,7588

0,7575

0,8837

6

$\mathbf{0 , 7 0 5 5}$

0,6824

0,6401

0,691

0,5774

0,6989

0,6848

6

$\mathbf{0 , 8 3 0 1}$

0,7929

0,8399

0,7703

0,8064

0,7705

0,8268

6

0,7571

0,7162

0,715

0,723

0,7256

0,7213

0,7313
La fiabilidad test-retest se comprobó sobre 138 pacientes que no habían mostrado cambios de su estado de salud general entre los dos controles. El coeficiente interclase obtenido fue de 0,92 para la puntuación global y superior a 0,70 para cada una de las dimensiones (Tabla 3).

El análisis de la validez convergente del cuestionario mostró una correlación moderada-alta y significativa entre la dimensión Función Sexual del cuestionario UCLA-PCI y la dimensión Vida Sexual del cuestionario CAVIPRES $(r=0,77)$. Para el resto de dimensiones, que no compartían contenidos, aunque se hallaron algunas correlaciones significativas, fueron débiles (Tabla 4).
Entre el cuestionario CAVIPRES y el SF-36, mostraron correlaciones moderadas y significativas las dimensiones Aspectos Psicológicos (r=0,53), Esperanza y futuro $(\mathrm{r}=0,52)$ del cuestionario CAVIPRES, con la Función Mental del SF-36, así como la puntuación total de CAVIPRES $(\mathrm{r}=0,57)$ y Función Mental del SF-36. Las dimensiones Esperanza $y$ Futuro $(\mathrm{r}=0,42)$ y la puntuación total del CAVIPRES $(r=0,41)$ mostraron una correlación modera-baja y significativa con la Función Física del SF-36 (Tabla 4).

La comparación de subgrupos de población determinados por aquellas características basales que, a priori, podría considerarse factores modifica- 


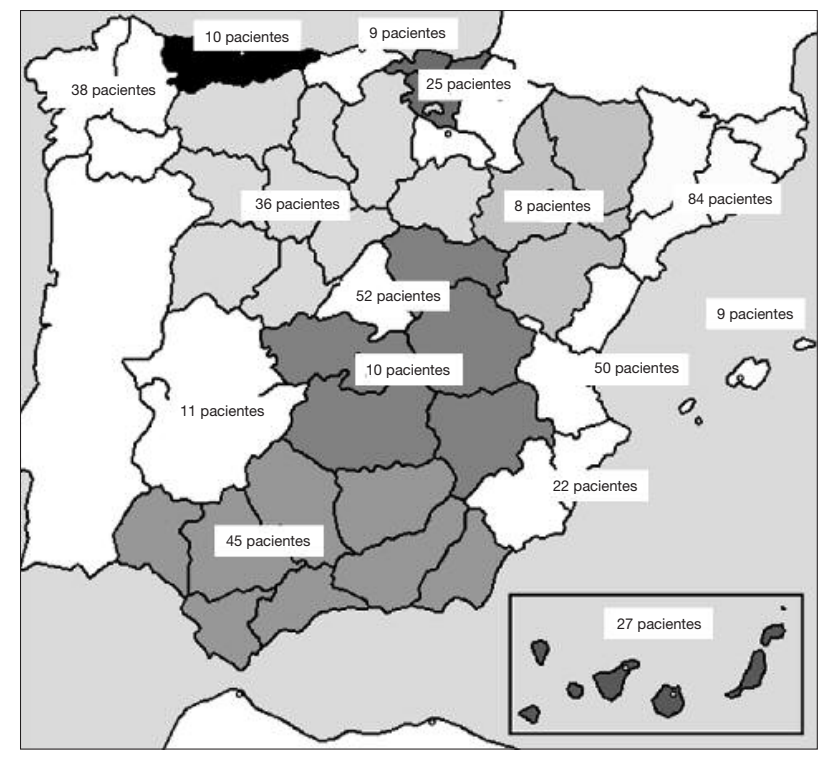

FIGURA 2. Distribución de los pacientes encuestados en fase III: Validación del cuestionario CAVIPRES-30 ( $n=436)$.

dores de la calidad de vida permitió explorar la validez de constructo (Tabla 5). La población con pareja estable presentaban puntuaciones del CAVIPRES-30 significativamente más altas en la dimensión Aspectos psicológicos y en Comunicación e información $(\mathrm{p}<0,001)$.
Los pacientes con 1-3 años trascurridos desde el diagnóstico del tumor mostraban mejores puntuaciones en Esperanza y futuro que aquellos en los que no había transcurrido más de un año y que aquellos en que ya había transcurrido más de 3 años desde el diagnóstico $(\mathrm{p}<0,01)$. Los pacientes con mayor tiempo de diagnóstico mostraban mayor impacto en Vida sexual, de acuerdo a las puntuaciones significativamente más bajas para esta dimensión ( $\mathrm{p}<0,01)$. De forma global, sin embargo, los pacientes con mayor tiempo transcurrido desde el diagnóstico mostraban puntuaciones significativamente más elevadas del CAVIPRES-30 (Tabla 5).

Los pacientes que estaban en fase de seguimiento mostraban mejor calidad de vida, de acuerdo con la puntuación global del CAVIPRES-30, que aquellos que estaban en fase de tratamiento $(\mathrm{p}=0,03)$. Para las subescalas de Aspectos psicológicos, Esperanza y futuro, Vida Sexual y Comunicación e información, también se hallaron diferencias significativas en este sentido $(\mathrm{p}<0,01)$ (Tabla 5).

Los pacientes que estaban recibiendo tratamiento sintomático para el dolor presentaban una puntuación global del CAVIPRES-30 significativamente más baja que los pacientes que no lo precisaban. Estas diferencias se mantenían también significativas para

Tabla 3. Distribución de puntuaciones, efectos suelo y techo, consistencia interna y fiabilidad test-retest del cuestionario CAVIPRES$30(\mathrm{~N}=436)$

\begin{tabular}{|c|c|c|c|c|c|c|}
\hline CAVIPRES-30 & Total & A.P. & E. y F. & v. s. & A. S. y P. & C. e I. \\
\hline Ítems (n) & 30 & 8 & 4 & 6 & 6 & 6 \\
\hline Media & 61,3 & 11,5 & 10,5 & 5,6 & 20 & 13,5 \\
\hline $\mathrm{DE}$ & 19,9 & 3,8 & 2,9 & 3,1 & 4,1 & 3,7 \\
\hline Rango teórico & $30-150$ & $8-40$ & $4-20$ & $6-30$ & $6-30$ & $6-30$ \\
\hline Rango observado & $33-133$ & $8-20$ & $4-15$ & $6-15$ & $7-25$ & $6-25$ \\
\hline E. Suelo ${ }^{1}(\%)$ & $0 \%$ & $1,2 \%$ & $0,2 \%$ & $31,8 \%$ & $0 \%$ & $1,6 \%$ \\
\hline E. Techo $2(\%)$ & $0 \%$ & $1,4 \%$ & $7,9 \%$ & $0,9 \%$ & $7,8 \%$ & $1,2 \%$ \\
\hline $\begin{array}{l}\text { Respuestas perdidas por paciente, } \\
\text { media(DE) }\end{array}$ & $0,35(1,8)$ & $0,06(0,6)$ & $0,02(0,24)$ & $0,06(0,5)$ & $0,2(0,7)$ & $0,05(0,5)$ \\
\hline $\begin{array}{l}\text { Tiempo de administración, N(\%) } \\
\text { Entre } 10 \text { y } 15 \mathrm{~min} . \\
\text { Entre } 15 \text { y } 20 \mathrm{~min} . \\
\text { Entre } 20 \text { y } 25 \mathrm{~min} . \\
\text { Más de } 30 \mathrm{~min} .\end{array}$ & $\begin{array}{c}139(31,9) \\
149(34,2) \\
94(21,6) \\
54(12,4)\end{array}$ & np & $\mathrm{np}$ & $\mathrm{np}$ & $\mathrm{np}$ & $\mathrm{np}$ \\
\hline $\begin{array}{l}\text { Cuestionario 'moderadamente' } \\
\text { - 'muy' fácil de responder, } \mathrm{N}(\%)\end{array}$ & $298(68,8)$ & $\mathrm{np}$ & np & $\mathrm{np}$ & $\mathrm{np}$ & $\mathrm{np}$ \\
\hline $\begin{array}{l}\text { Alfa de Cronbach } \\
\mathrm{CCI}^{3}(\mathrm{n}=138)\end{array}$ & $\begin{array}{l}0,77 \\
0,92\end{array}$ & $\begin{array}{l}0,91 \\
0,83\end{array}$ & $\begin{array}{l}0,84 \\
0,84\end{array}$ & $\begin{array}{l}0,92 \\
0,83\end{array}$ & $\begin{array}{l}0,76 \\
0,86\end{array}$ & $\begin{array}{l}0,81 \\
0,83\end{array}$ \\
\hline
\end{tabular}

${ }^{1}$ Porcentaje de pacientes con la peor puntuación posible; ${ }^{2}$ Porcentaje de pacientes con la mejor puntuación posible; ${ }^{3}$ CCI: Coeficiente de Correlación Intraclase. Estabilidad evaluada por el propio paciente; np: no procede. A.P: Aspectos psicológicos; E. y F: Esperanza y futuro; V. D: Vida sexual; A.S. y P: Apoyo social y pareja; C. e I: Comunicación e información. 
Tabla 4. Validez convergente del cuestionario CAVIPRES-30, con los cuestionarios UCLA-PCI y SF-36 (Coeficientes de correlación de Pearson) (N=436)

\begin{tabular}{lcccccc}
\hline & Total & A.P. & E. y F. & V. S. & A. S. y P. & C. e I. \\
\hline UCLA-PCI & & & & & & \\
Función Urinaria & 0,23 & 0,19 & 0,17 & 0,18 & 0,14 & 0,11 \\
$\begin{array}{l}\text { Función Deposición } \\
\text { Función Sexual }\end{array}$ & 0,35 & 0,35 & 0,24 & 0,16 & 0,12 & 0,27 \\
SF-36 & 0,39 & 0,10 & 0,30 & 0,77 & 0,16 & $<0,1$ \\
Función Mental & 0,57 & 0,53 & 0,52 & 0,16 & 0,23 & 0,37 \\
Función Física & 0,41 & 0,33 & 0,42 & 0,29 & 0,13 & 0,15 \\
\hline
\end{tabular}

A.P: Aspectos psicológicos; E. y F: Esperanza y futuro; V. D: Vida sexual; A.S. y P: Apoyo social y pareja; C. e I: Comunicación e información.

las subescalas Aspectos psicológicos, Esperanza y futuro, Vida Sexual y Apoyo social y pareja $(\mathrm{p}<0,01)$ entre ambos subgrupos. Resultados semejantes se obtuvieron al comparar pacientes que estaban recibiendo tratamiento sintomático con aquellos que no lo precisaban (Tabla 5).

Los pacientes que referían que su estado de salud en general había mejorado respecto a la visita basal de forma sustancial mostraron un tamaño del efecto pequeño para la puntuación global de CAVIPRES-30. Sin embargo, los pacientes que re- ferían mejoría sustancial, mostraban cambios de puntuación importantes en las subescalas de Esperanza y futuro y Apoyo social y pareja, y un cambio de puntuación muy cuantioso en Vida Sexual, lo que determinó un tamaño del efecto muy grande para estas 3 dimensiones y en especial para la última mencionada (Tabla 6).

\section{DISCUSIÓN}

\section{Desarrollo del cuestionario CAVIPRES}

El desarrollo y validación del primer cuestionario español para la evaluación de la calidad de vida percibida por en pacientes con CaP se llevó a cabo siguiendo rigurosamente la metodología de validación de instrumentos psicométricos ${ }^{20,21} \mathrm{y}$ de acuerdo con las recomendaciones internacionales en esta materia ${ }^{23,24}$.

La búsqueda bibliográfica inicial evidenció que hay pocos instrumentos desarrollados específica-

Tabla 5. Validez de grupos conocidos del cuestionario CAVIPRES-30, según las características del paciente (N=436)

\begin{tabular}{|c|c|c|c|c|c|c|}
\hline Cavipres-30 & Total & A.P. & E. y F. & v. $\mathbf{S}$. & A. S. y P. & C. e I. \\
\hline \multicolumn{7}{|c|}{ Pareja estable, N(\%) } \\
\hline Sí & $62,4(10,4)$ & $11,6(3,9)$ & $10,6(2,8)$ & $5,7(3,1)$ & $20,7(3,4)$ & $13,6(3,7)$ \\
\hline No & $55,1(11,6)$ & $11,3(3,6)$ & $10,1(3)$ & $5(2,9)$ & $15,9(5)$ & $13,2(3,6)$ \\
\hline p-valor & 0,364 & 0,000 & 0,557 & 0,155 & 0,078 & 0,000 \\
\hline \multicolumn{7}{|c|}{ Tiempo desde el diagnóstico de tumor primario, media (DE) } \\
\hline Hasta 1 año & $60,6(10,4)$ & $11,1(3,5)$ & $10,1(2,7)$ & $6,0(3,3)$ & $20,1(3,9)$ & $12,9(3,5)$ \\
\hline Entre 1-3 años & $61,4(11,3)$ & $11,5(4,3)$ & $11(2,8)$ & $5,4(2,9)$ & $20(4,1)$ & $13,7(3,8)$ \\
\hline Más de 3 años & $62,7(11,5)$ & $12,8(3,8)$ & $10,8(2,9)$ & $4,6(2,5)$ & $19,4(4,2)$ & $14,8(3,9)$ \\
\hline p-valor & 0,000 & 0,338 & 0,002 & 0,011 & 0,001 & 0,417 \\
\hline \multicolumn{7}{|c|}{ Opción terapéutica, N(\%) } \\
\hline En seguimiento & $66(11,7)$ & $12,9(4,2)$ & $11,6(2,9)$ & $6,1(3,1)$ & $20,9(3,7)$ & $14,3(4,1)$ \\
\hline En tratamiento & $59,9(10,3)$ & $11,1(3,6)$ & $10,2(2,7)$ & $5,4(3,1)$ & $19,7(4,1)$ & $13,3(3,5)$ \\
\hline $\mathrm{p}$-valor & 0,030 & 0,000 & 0,000 & 0,000 & 0,063 & 0,013 \\
\hline \multicolumn{7}{|c|}{ Tratamiento sintomático debido al Dolor, $\mathbf{N}(\%)$} \\
\hline Con tratamiento & $53,1(8)$ & $9,6(3,6)$ & $8,4(2,2)$ & $4(1,9)$ & $18,9(3,9)$ & $12,1(2,9)$ \\
\hline Sin tratamiento & $61,5(10,3)$ & $11,5(3,6)$ & $10,5(2,7)$ & $5,6(3,2)$ & $19,9(4,1)$ & $13,6(3,7)$ \\
\hline $\mathrm{p}$-valor & 0,005 & 0,000 & 0,000 & 0,000 & 0,000 & 0,098 \\
\hline \multicolumn{7}{|c|}{ Tratamientos de tipo sintomático, $\mathbf{N}(\%)$} \\
\hline Con tratamiento & $57,3(9,9)$ & $10,4(3,5)$ & $9,6(2,7)$ & $4,7(2,6)$ & $19,5(4,1)$ & $12,8(3,3)$ \\
\hline Sin tratamiento & $62,1(10,4)$ & $11,8(3,6)$ & $10,6(2,7)$ & $5,9(3,3)$ & $19.9(4)$ & $13,8(3,7)$ \\
\hline p-valor & 0,009 & 0,000 & 0,000 & 0,001 & 0,000 & 0,419 \\
\hline
\end{tabular}

A.P: Aspectos psicológicos; E. y F: Esperanza y futuro; V. D: Vida sexual; A.S. y P: Apoyo social y pareja; C. e I: Comunicación e información. 
Tabla 6. Sensibilidad al cambio del cuestionario CAVIPRES-30, entre los pacientes que reportan una mejora* en el estado de salud $(\mathrm{N}=96)$

\begin{tabular}{|c|c|c|c|c|c|c|}
\hline $\begin{array}{l}\text { CAVIPRES-30 } \\
\text { puntuación }\end{array}$ & Total & A.P. & E. $\mathbf{y}$ F. & V. S. & A. S. y P. & C. e I. \\
\hline Visita 1, media (DE) & $61,8(10,3)$ & $11,3(3,8)$ & $10,8(2,8)$ & $5,6(3,1)$ & $20,8(4)$ & $13,2(3,7)$ \\
\hline Visita 2, media (DE) & $64,6(9,4)$ & $11,5(2,4)$ & $5,2(2,8)$ & $21,4(3,6)$ & $13,6(3,8)$ & $13(3,4)$ \\
\hline p-valor (entre visitas) & 0,003 & 0,000 & 0,011 & 0,202 & 0,071 & 0,176 \\
\hline Tamaño del efecto & 0,27 & 0,06 & 1,97 & 5,03 & 1,8 & 0,06 \\
\hline
\end{tabular}

A.P: Aspectos psicológicos; E. y F: Esperanza y futuro; V. D: Vida sexual; A.S. y P: Apoyo social y pareja; C. e I: Comunicación e información (*): Mejora: pacientes que respondieron con puntuaciones de 1 a 3 a la pregunta: En estos últimos 3 meses, ¿cree que su estado de salud es..?, 1= "ha mejorado muchísimo", 2= "ha mejorado mucho", 3="ha mejorado bastante"......13="ha empeorado muchísimo"

mente para medir calidad de vida en CaP. Los instrumentos específicos ya existentes eran el UCLAPCI y el EPIC, este último todavía no había sido validado al español cuando se inició este proyecto ${ }^{25}$ Ambos cuestionarios recogen información sobre el impacto del CaP en la función sexual, función urinaria y digestiva, y en el caso de EPIC también en la función hormonal. Sin embargo, la discusión con expertos y los grupos focales con pacientes realizados durante el desarrollo de CAVIPRES expusieron ciertos aspectos derivados de estas afectaciones que no podían ser detectados por dichos cuestionarios. El cuestionario CAVIPRES incluye la dimensión Apoyo social y pareja que ahonda en el impacto del $\mathrm{CaP}$ en el entorno inmediato del paciente y la dimensión Aspectos psicológicos que recoge información de cómo percibe el paciente tal impacto. Mientras que, tanto el UCLA-PCI como el EPIC sólo contienen un ítem general en cada una de las funciones que permita medir cómo afecta la alteración de tal función en la calidad de vida del paciente.

Desde el momento del diagnóstico del cáncer y durante el tiempo de supervivencia, el sujeto cambia su visión del mundo, sus prioridades y su forma de asumir responsabilidades y esto afecta a su calidad de vida ${ }^{26}$. En pacientes ancianos con $\mathrm{CaP}$, se ha visto que las expectativas de vida más largas se asocian a un mejor estado físico y psicológico ${ }^{27}$. Por tanto, es crucial que los facultativos sean capaces de detectar aspectos relacionados con la esperanza de vida del paciente para facilitar un soporte psicológico adecuado a éste y a su entorno. CAVIPRES-30 incluye una dimensión destinada a evaluar percepciones del paciente relacionadas con sus expectativas de vida frente a la evolución de su enfermedad (Esperanza y futuro), característica que lo convierte en un cuestionario totalmente original respecto a otros cuestionarios específicos para $\mathrm{CaP}$ ya existentes.
En la actualidad, los profesionales médicos son cada vez más conscientes de que la participación del paciente, debidamente informado, en la elección de su tratamiento supone un pacto en la decisión terapéutica que mejora la satisfacción del paciente; hecho que también incide en su calidad de vida. La opción del paciente de aceptar terapias más agresivas a cambio de mínimos beneficios ${ }^{2,3}$ o terapias más suaves a cambio de una mejor calidad de vida ${ }^{4}$, sólo puede darse cuando el médico conoce la voluntad del paciente sin que interfieran factores cognitivos o afectivos.

CAVIPRES-30 puede constituir un instrumento útil para medir aspectos relacionados con la decisión terapéutica ya que contiene una serie de ítems agrupados como Comunicación e información que abordan aspectos de la información recibida/deseada sobre la propia enfermedad.

\section{Validación del cuestionario CAVIPRES}

El cuestionario CAVIPRES-30 resultó ser un instrumento con una buena aceptabilidad y el análisis de sus propiedades psicométricas demuestra su factibilidad, validez, fiabilidad y sensibilidad al cambio.

Que la mayoría de pacientes no requiriera más de 20 minutos para completar el cuestionario (76\%) y que lo consideraran "fácil" de responder (69\%) lo define como instrumento ágil y factible de ser autoadministrado.

El cuestionario obtuvo un bajo nivel de no respuesta y cabe mencionar que la dimensión Vida sexual, cuyos ítems podrían haber motivado una cierta abstención en la respuesta, no resultó ser la dimensión con mayor porcentaje de pérdidas.

Para el CAVIPRES global y para cada una de sus dimensiones se podía rechazar el "efecto techo" y el "efecto suelo" dado que menos del 15\% de los encuestados alcanzaron la máxima y la mínima puntuación 
posible, respectivamente ${ }^{28}$. En la dimensión Vida sexual, sí se detectó un efecto suelo $(31,8 \%)$, aunque éste es un problema habitual y compartido por muchos de los instrumentos que miden disfunción sexual $^{29,30}$.

El cuestionario es altamente fiable, de acuerdo con el coeficiente alfa de Cronbach obtenido $(r=0,77)$ para la puntuación global y para cada una de sus dimensiones. Lo que demuestra una consistencia interna muy satisfactoria, superior a los estándares psicométricos $^{23}$. Además, CAVIPRES-30 mostró una elevada reproducibilidad test-retest, obteniendo un coeficiente interclase de 0,92, superior al CCI que se acepta como fiable la puntuación de un cuestionario ${ }^{22}$.

Las altas correlaciones halladas entre la subescala Vida sexual del CAVIPRES y Función sexual del UCLA-PCI están en consonancia con las hipótesis de trabajo del estudio en fase de validación y prueba que estas dimensiones en sendos cuestionarios miden los mismos conceptos. También guarda coherencia que los dimensiones de Función urinaria y Función de Deposición del UCLA-PCI mostraran bajas correlaciones con la puntuación total del CAVIPRES así como para sus diferentes dimensiones, ya miden aspectos dispares.

La correlaciones moderadas entre la Función mental del SF-36 y las dimensiones Aspectos psicológicos y Esperanza y futuro de CAVIPRES sugieren que la mejor calidad de vida en general, se asocia a mejores expectativas de vida y mejor estado psicológico del paciente con CaP. Aunque esta afirmación es coherente y consistente, de nuevo, es preciso reconocer los matices de esta asociación: las dimensiones que se analizan procedentes del CAVIPRES y del SF-36, si bien incluyen ítems con contenidos semejantes, su enfoque es diferente. No es de extranar que las dimensiones Vida Sexual, Apoyo social y pareja y Comunicación e Información, correlacionaran de forma muy pobre con la Función mental y Función Física del SF-36.

La comparación de la puntuación de CAVIPRES entre subgrupos definidos aportó la información necesaria para sostener que el cuestionario gozaba de validez de constructo. Se obtuvieron mejores puntuaciones en global y para las dimensiones como Esperanza y futuro, Vida sexual y Apoyo social y pareja en pacientes con pareja estable, lo que corrobora la idea, a priori, de que el apoyo de la pareja mejora la calidad de vida en el paciente con $\mathrm{CaP}$.
También las respuestas obtenidas en función del tiempo desde el diagnóstico correspondían con los resultados en calidad de vida esperables. Globalmente, los pacientes con mayor tiempo de diagnóstico presentaban mayor calidad de vida. Sin embargo, de forma particular esto no se reproducía en todas las dimensiones que mostraban diferencias significativas: el diagnostico más reciente implicaba menos expectativas de vida pero mejor vida sexual. Esto se explicaría considerando que el paciente con un tiempo de diagnóstico inferior a 1 año ha tenido menos tiempo para asumir el diagnostico, lo que se traduce en deterioro de su calidad de vida relacionada con Esperanza y futuro. Del mismo modo, este tipo de paciente probablemente sería más joven y con un proceso oncológico menos avanzado, lo que justifica sus mejores puntuaciones en Vida sexual.

Las peores puntuaciones en Aspectos psicológicos, Esperanza y futuro y Vida sexual en pacientes en fase de tratamiento respecto a pacientes en seguimiento podrían estar relacionadas con las molestias y efectos secundarios derivados del tratamiento oncológico. El dolor en el paciente oncológico es reconocido como el síntoma más deletéreo en su calidad de vida y así lo corroboraron los resultados mostrando peores puntuaciones del CAVIPRES en el paciente en tratamiento sintomático del dolor. Del mismo modo, los síntomas propios del CaP, tales como disfunción urinaria, impotencia, dolor, etc, tienen un grave impacto en la calidad de vida del paciente ${ }^{5,6}$, y como era de esperar, aquellos pacientes que no precisaban tratamiento sintomático mostraron mejores puntuaciones del CAVIPRES.

Finalmente, el análisis de sensibilidad al cambio también ofreció resultados satisfactorios en cuanto a que se detectó un tamaño del efecto aceptable para la puntuación global $(<0,2)$ y pequeño, que es lo que cabía esperar para un intervalo de tiempo no superior a 3 meses entre las dos administraciones del cuestionario.

Recientemente se ha realizado la validación lingüística al español el cuestionario $\mathrm{EPIC}^{25}$, como un instrumento fiable, válido y sensible al cambio para valorar calidad de vida en pacientes con CaP. Los autores de la validación reconocen como una posible limitación el hecho de que se administrara mediante entrevista telefónica, por los que sus resultados no serían generalizables a la autoadministración, como es el caso de CAVIPRES-30 que fue cumplimentado por el propio paciente. Por otra 
parte, las poblaciones encuestadas no son equiparables, debido a que el $62 \%$ de pacientes del estudio EPIC presentaban CaP localizado y el estudio CAVIPRES incluyó pacientes con estadios más avanzados del tumor.

\section{CONCLUSIONES}

Las diferentes fases del proyecto CAVIPRES han logrado desarrollar y validar una herramienta manejable, fiable y sensible, específica para evaluar calidad de vida en el paciente con CaP. La rigurosa metodología empleada en su desarrollo ha permitido conseguir un cuestionario original por que incorpora aspectos novedosos en la medida de la calidad de vida del paciente con CaP.

Por características psicométricas, CAVIPRES-30 es un cuestionario apto para ser utilizado en la investigación clínica.

\section{Agradecimientos}

Este proyecto ha sido posible gracias al patrocinio de Abbott Laboratories S.A. Los autores agradecen la colaboración de Rous de Castellar en la preparación del manuscrito, de Verónica Miranda en la coordinación técnica (3D-Health Research) y de Carlos Campo (Abbott Laboratorios).

Agradecimientos a todos los urólogos que han participado en el desarrollo del Estudio Cavipres.

\section{REFERENCIAS}

1. Litwin MS: Measuring health-related quality of life in men with prostate cancer. J Urol 1994; 152:1882-1887.

2. Slevin ML, Stubbs L, Plant HJ, Wilson P, Gregory WM, Armes PJ, Downer SM. Attitudes to chemotherapy: comparing views of patients with cancer with those of doctors, nurses, and general public. BMJ. 1990;300(6737):1458-1460.

3. Jansen SJ, Kievit J, Nooij MA, de Haes JC, Overpelt IM, van Slooten H, Maartense E, et al. Patients' preferences for adjuvant chemotherapy in earlystage breast cancer: is treatment worthwhile? Br J Cancer. 2001;84(12): 1577-1585.

4. Van Tol-Geerdink JJ, Stalmeier PF, van Lin EN, Schimmel EC, Huizenga H, van Daal WA, Leer JW. Do patients with localized prostate cancer treatment really want more aggressive treatment?J Clin Oncol. 2006;24(28):4581-4586.

5. FJ, Barry MJ, Lu-Yao G et al: Effect of radical prostatectomy for prostate cancer on patients quality of life: Results from a Medicare survey. Urology 1995; 45: 1007-1015.

6. Gburek B, Harmon B, Chodak GW: Quality of life assessment in patients with prostate cancer treated by radical prostatectomy, radiation therapy or a combination. J Urol; 1992; 147: 466A

7. Litwin MS, Hays RD, Fink A, Ganz PA, Leake B, Leach GE, Brook RH. Quality of life outcomes in men with localized prostate cancer. JAMA 1995;273:129-135.

8. McHorney CA, Ware JE Jr, Raczek AE. The MOS 36-Item Short-Form Health Survey (SF-36): II. Psychometric and clinical tests of validity in measuring physical and mental health constructs. Med Care. 1993;31(3):247-263.

9. Hays RD, Sherbourne CD, Mazel RM. The RAND 36-Item Health Survey 1.0. Health Econ 1993;2(3):217-227.

10. Wei JT, Dunn RL, Litwin MS, Sandler HM, Sanda MG. Development and validation of the expanded prostate cancer index composite (EPIC) for comprehensive assessment of health-related quality of life in men with prostate cancer. Urology 2000;56(6):899-905.
11. Esper P, Mo F, Chodak G, Sinner M, Cella D, Pienta KJ. Measuring quality of life in men with prostate cancer using the functional assessment of cancer therapy-prostate instrument. Urology 1997;50(6):920-928.

12. Bjordal K, Kaasa S. Psychometric validation of the EORTC core quality of life questionnaire , 30 item version and a diagnosis specific module for head and neck cancer patients. Acta Oncol 1992;31: 311-321.

13. Van Andel G, Bottomley A, Fossa S, Efficace F, Coens C, Guerif S, Kynaston $\mathrm{H}$, et al. on behalf of the EORTC Genito-Urinary Tract Cancer Group and the EORTC Quality of Life Group. An international field study of the EORTC QLQ-PR25: A questionnaire for assessing the health-related quality of life of patients with prostate cancer. Eur J Cancer 2008;44(16): 2418-2424.

14. Ferrer M, Garin O, Pera J, Prats JM, Mendivil J, Alonso J, De Paula B, et al. Grupo Multicéntrico Español de Cáncer de Próstata Localizado. Evaluación de la calidad de vida de los pacientes con cáncer de próstata localizado: validación de la versión española del cuestionario EPIC. Med Clin (Barc) 2009; 132(4):128-135.

15. Arraras JI, Manterola A, Dominguez MA, Arias F, Villafranca E, Romero P, Martínez E, et al. Impact of radiotherapy on the quality of life of elderly patients with localized breast cancer. A prospective study. Clin Transl Oncol. 2008;10(8):498-504.

16. Wild D, Grove A, Martin M, Eremenco S, McElroy S, Verjee-Lorenz A, Erikson P; ISPOR Task Force for Translation and Cultural Adaptation. Principles of Good Practice for the Translation and Cultural Adaptation Process for Patient-Reported Outcomes (PRO) Measures: report of the ISPOR Task Force for Translation and Cultural Adaptation. Value Health 2005;8(2):94-104.

17. Herdman M, Fox-Rushby J, Badia X. A model of equivalence in the cultural adaptation of HRQoL instruments: the universalist approach. Qual Life Res 1998;7:323-335.

18. Alonso J, Prieto L, Antó JM. La versión española del SF-36 Health Survey (Cuestionario de Salud SF-36): un instrumento para la medida de los resultados clínicos. Med Clin (Barc) 1995;104(20):771-776.

19. Krongrad, A; Perczek, RE; Burke, MA, et al. Reliability of Spanish translations of select urological quality of life instruments. J Urol 1997;158:493-496.

20. Nunnally J, Bernstein IH. Psychometric Theory, 3rd ed. New York: McGrawHill; 1994.

21. Streiner DL, Norman GR (2003). Health measurement scales: a practical guide to their development and use. New York: Oxford University Press.

22. Hays RD, Anderson R, Revicki D. Psychometric considerations in evaluating health-related quality of life measures. Qual Life Res 1993;2(6):441-449.

23. Scientific Advisory Committee of the Medical Outcomes Trust. Assessing health status and quality-of-life instruments: attributes and review criteria Qual Life Res 2002;11(3):193-205.

24. US Department of Health and Human Services. 2006. Guidance for industry patient-reported outcome measures: use in medical product development to support labeling claims. (consultado: 16/03/2009) Disponible en: http://www.fda.gov/ cber/gdlns/prolbl.htm

25. Ferrer M, Garin O, Pera J, Prats JM, Mendivil J, Alonso j et al. Evaluación de la calidad de vida de los pacientes con cáncer de próstata localizado. Validación de la versión española del cuestioanario EPIC. Med Clin 2009; 132(4): 128-135.

26. Zebrack BJ. Cancer survivor identity and quality of life. Cancer Pract. 2000 8(5):238-242.

27. Diefenbach M, Mohamed NE, Horwitz E, Pollack A. Longitudinal associations among quality of life and its predictors in patients treated for prostate cancer: the moderating role of age. Psychol Health Med. 2008;13(2):146-61.

28. McHorney CA, Tarlov AR: Individual-patient monitoring in clinical practice: are available health status surveys adequate? Qual Life Res 1995;4(4):293 307.

29. Bobes J, González MP, Bascarán MT, Clayton A, Garcia M, Rico-Villa de Moros F, Banús S. Evaluating changes in sexual functioning in depressed patients: sensitivity to change of the CSFQ. J Sex Marital Ther 2002;28(2):93103.

30. Moinpour CM, Darke AK, Donaldson GW, Thompson IM Jr, Langley C, Ankerst DP, Patrick DL, et al. Longitudinal analysis of sexual function reported by men in the Prostate Cancer Prevention Trial.J Natl Cancer Inst 2007;99(13):1025-1035.

Correspondencia autor: Dr. Francisco Gómez Veiga

Complej Hospitalario Universitario A Coruña

Xubias de Arriba, 84 - 15006 A Coruña

Tel.: 981178000

E-mail autor: fgveiga@telefonica.net

Información artículo: Original - Cáncer de próstata

Trabajo recibido: marzo 2009

Trabajo aceptado: abril 2009 